\title{
Protection of corneal endothelium from irrigation damage: a comparison of sodium hyaluronate and hydroxypropylmethyl- cellulose
}

H.S. Dua

Department of

Ophthalmology

Queen's Medical Centre

University Hospital

Nottingham, UK

D.A. Benedetto

Wills Eye Hospital Jefferson Medical College

Philadelphia, USA

\section{A. Azuara-Blanco}

Princess Alexandra Eye

Pavilion

Edinburgh, UK

\section{Professor}

Harminder S. Dua

B-Floor, South Block

Queen's Medical Centre

Nottingham NG7 2UH, UK

Tel: +44 (0)1159709796

Fax: +44 (0)1159709963

e-mail:

Harminder.Dua@nottingham.ac.uk

Commercial and proprietary interest: None

This study was supported by a grant from

Storz Ophthalmics

Received: 18 February 1999 Accepted in revised form: 25 August 1999

\begin{abstract}
Purpose To compare the endothelial protection of sodium hyaluronate and hydroxypropylmethylcellulose against endothelial damage induced by irrigation. Methods An in vitro assay with freshly excised porcine eyes was developed using the Janus green photometry technique. Irrigation and aspiration technique was standardised. Forty pairs of porcine eyes were used. One randomly chosen eye was filled with sodium hyaluronate (SH) and the other with hydroxypropylmethylcellulose (HPMC). Irrigation and aspiration was carried out with balanced salt solution for $5 \mathrm{~min}$. Twenty additional pairs of porcine eyes served as controls. Student's $t$-test was used for statistical analysis.

Results Both viscoelastic agents protected the endothelium as compared with controls. The endothelial protection, determined with the Janus green photometric technique, was significantly greater with HPMC than with SH.

Conclusions Viscoelastic agents are effective in protecting the endothelium from irrigation damage in porcine eyes in vitro. HPMC provided greater protection than $\mathrm{SH}$ in this particular model.
\end{abstract}

Key words Cataract, Cornea, Endothelium, Irrigation-aspiration, Janus green, Viscoelastics

One of the most important functions of viscoelastic solutions in ophthalmology is protection of the corneal endothelium during cataract surgery. The corneal endothelium can be accidentally damaged by instrument or intraocular lens touch, by lens fragments and air bubbles during phacoemulsification and by irrigating solutions used during routine irrigation and aspiration. ${ }^{1-3}$ All the viscoelastic
HARMINDER S. DUA,

DOMINICK A. BENEDETTO,

AUGUSTO AZUARA-BLANCO agents presently in use possess sufficient viscosity at rest to provide a mechanical barrier from intraocular lens and instrument touch of the endothelium. Hyaluronic acid based viscoelastics, such as sodium hyaluronate of high molecular weight ( $\mathrm{SH}$; Healon, Pharmacia) and sodium hyaluronate of lower molecular weight (Amvisc Plus, Iolab) provide protection under these circumstances because of their high viscosity at rest. ${ }^{3-6}$ With increased emphasis on the removal of cataract by phacoemulsification, the potential for damage from air bubbles, lens fragments and irrigating solutions is increased. This shift in technique has emphasised the endothelial coating properties of viscoelastic solutions.

From a clinical standpoint viscoelastic agents can be divided into two broad categories relating to gross endothelial viscoelastic coating. $\mathrm{SH}$ is an example of the first category, which do not provide a visible endothelial coating during surgery and are easily aspirated from the anterior chamber. In the second category, viscoelastics such as

hydroxypropylmethylcellulose (HPMC; Occucoat, Storz) and chondroitin sulphate-sodium hyaluronate (Viscoat, Alcon) produce a visible endothelial coating and are less easily aspirated.?

Studies have documented, at least in the short post-operative period, greater endothelial protection from solutions producing grossly visible endothelial coatings during ultrasonic agitation of the anterior chamber with air bubbles. ${ }^{3}$ It has also been noted that irrigating solutions can cause endothelial damage. ${ }^{8}$

In this study we describe the use of the Janus green photometry technique ${ }^{9}$ to quantify the difference in endothelial damage occurring during irrigation and aspiration (I\&A) of the anterior chamber of porcine eyes with and without HPMC or SH. 


\section{Materials and methods}

\section{Janus green standard curve}

The Janus green photometric technique was used to assess endothelial damage. ${ }^{9}$ Twenty serial dilutions of Janus green (Sigma) ranging from $0.5 \mathrm{mg} / 1$ to $20 \mathrm{mg} / 1$ were made in absolute alcohol. Samples corresponding to each concentration were read in a photometer at $650 \mathrm{~nm}$. Extinction values of each sample were plotted against their respective concentrations to give the Janus green standard curve

\section{Janus green staining and elution}

Porcine eyes were used in this study. Freshly excised pig eyes from 9- to 12-month-old animals were obtained from an abattoir (Hatfield Meat Packing Co.). Each pair of eyes was placed in a separate bottle and transported on ice. The eyes were processed within $60-150 \mathrm{~min}$ of enucleation. Corneoscleral buttons with a $2 \mathrm{~mm}$ scleral rim were excised, taking care not to damage the endothelium. All excisions were done by the same investigator (H.S.D.). The corneoscleral buttons were rinsed in a sterile balanced salt solution (BSS, Alcon Laboratories, Fort Worth, TX) containing 0.05\% Tween20 , for $3 \mathrm{~min}$. They were then placed in a shallow wax cup with the epithelial surface against the wax. The endothelial surface was covered with $200 \mu$ l of a $1 \%$ solution of Janus green in BSS for $90 \mathrm{~s}$. Excess dye was tipped off and the corneoscleral button was rinsed in three changes of BSS for a total duration of $3 \mathrm{~min}$ A $9 \mathrm{~mm}$ button was punched out from the centre of the cornea using a punch trephine (H\&I Instrumentation, $\mathrm{NJ})$. The corneal button was placed in a test tube containing $1 \mathrm{ml}$ of absolute alcohol for $90 \mathrm{~s}$ to elute dye bound to the corneal button. The tube was vortexed and the extinction value of the solution read at $650 \mathrm{~nm}$.

\section{Standard endothelial damage}

Fifteen pairs of porcine eyes were processed to calculate Janus green extinction values for standard endothelial damage. The corneoscleral button obtained from one eye of each pair was directly stained with Janus green. Extinction values of this group were taken to represent ' $0.0 \%$ ' endothelial damage. Endothelium from the corneoscleral buttons obtained from the other eye of each pair was mechanically wiped off with moist cotton and then stained with Janus green. Extinction values of this group were taken to represent ' $100 \%$ ' endothelial damage. The extinction values corresponding to ' $0.0 \%$ ' and ' $100 \%$ ' damage were superimposed on the Janus green standard curve. Intermediate points corresponding to ' $25 \%$ ', ' $50 \%$ ' and ' $75 \%$ ' damage were also marked (Fig. 1).

Corneoscleral buttons obtained from a further 20 paired porcine eyes were subjected to ' $0.0 \%$ ' and ' $100 \%$ ' damage as described above. Each of 10 pairs of corneoscleral buttons was covered with SH for $5 \mathrm{~min}$ and the remaining 10 pairs covered with HPMC for $5 \mathrm{~min}$.

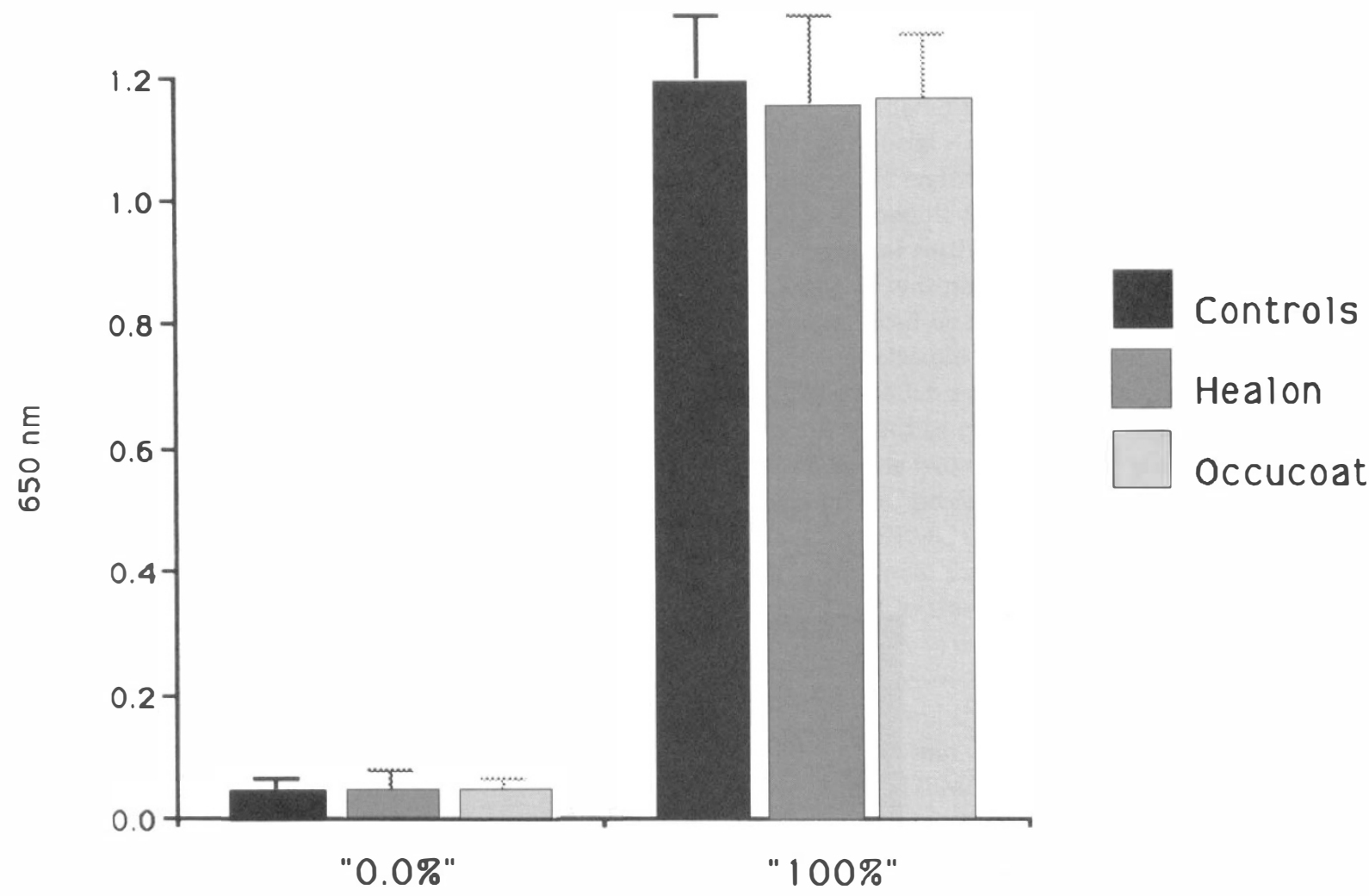

Fig. 1. Janus green photometry of corneoscleral buttons that were subjected to ' $0.0 \%$ ' and ' $100 \%$ ' endothelial damage prior to staining (mean values and standard deviation). Controls were corneoscleral buttons that were not pre-treated with SH (Healon) or HPMC (Occucoat). The Healon and Occucoat columns represent corneoscleral buttons that were pre-treated with Healon or Occucoat respectively for 5 min before staining. 
One corneoscleral button of each pair had ' $0.0 \%$ ' endothelial damage and the other had ' $100 \%$ ' endothelial damage. At the end of $5 \mathrm{~min}$ the corneoscleral buttons were processed for Janus green staining and elution as described above.

\section{Standardisation of irrigation and aspiration for optimal endothelial damage}

Porcine eyes were mounted on a stand and held in a clamp under a stereomicroscope. The clamp was tightened sufficiently to give an intraocular pressure of $15-18 \mathrm{mmHg}$. A standard $3 \mathrm{~mm}$ limbal incision was made and the anterior chamber irrigated and aspirated with BSS using a Cavitron/Kelman I\&A unit (Cooper Vision, Irvine, CA) with the switch in the 'IA $\mathrm{min}^{\prime}$ position, giving a flow rate of approximately $25 \mathrm{ml} / \mathrm{min}$. The tip of the I\&A canula was kept in the centre of the pupil in the plane of the iris with the irrigation ports directed horizontally. The anterior chambers were irrigated and aspirated for 3,5 and $8 \mathrm{~min}$. Ten pairs of eyes were used for each time point. One eye of each pair was subjected to I\&A while the other served as control. After I\&A, corneoscleral buttons were dissected and processed for Janus green staining and elution as described.

\section{Comparison of the protective effect of $\mathrm{SH}$ and HPMC against IEA-induced endothelial damage}

Forty pairs of eyes were used in this experiment. The anterior chamber of one eye of each pair, right or left picked at random, was filled with $0.25-0.3 \mathrm{ml}$ of $\mathrm{SH}$ and the other was filled with a similar volume of HPMC.
Irrigation and aspiration with BSS was carried out for $5 \mathrm{~min}$ as described. The corneoscleral buttons were dissected. $\mathrm{SH}$ was more readily rinsed off the corneoscleral buttons than HPMC when BSS was used as the rinsing solution but both washed off equally well when $0.05 \%$ Tween- 20 was added to the BSS solution and the buttons rinsed for $3 \mathrm{~min}$ before Janus green staining. The corneal buttons were then processed for Janus green staining and elution as described above. Addition of Tween-20 to the wash solution and rinsing for $3 \mathrm{~min}$ did not induce any further damage to the endothelium as assessed by Janus green staining. This was determined in pig eyes before including the use of Tween-20 in the experiment.

\section{Results}

The extinction values of the different concentrations of Janus green in absolute alcohol were plotted on a graph. The extinction values showed a progressive linear correlation with the concentration of Janus green. The mean value for ' $0.0 \%$ ' corneal endothelial damage was 0.04 and that for ' $100 \%$ ' damage was 1.17 (Fig. 1).

Control corneoscleral buttons in which ' $0.0 \%$ ' or ' $100 \%$ ' endothelial damage was produced and the surface covered with SH or HPMC for 5 min before Janus green staining and elution gave extinction values very similar to those where these substances were not used (Fig. 1).

Three minutes of I\&A with BSS produced an average of $19.6 \%$ corneal endothelial damage, $5 \mathrm{~min}$ of I\&A produced an average of $43.5 \%$ corneal endothelial damage and $8 \mathrm{~min}$ of I\&A produced an average of $49.5 \%$ corneal endothelial damage (Fig. 2). The mean Janus

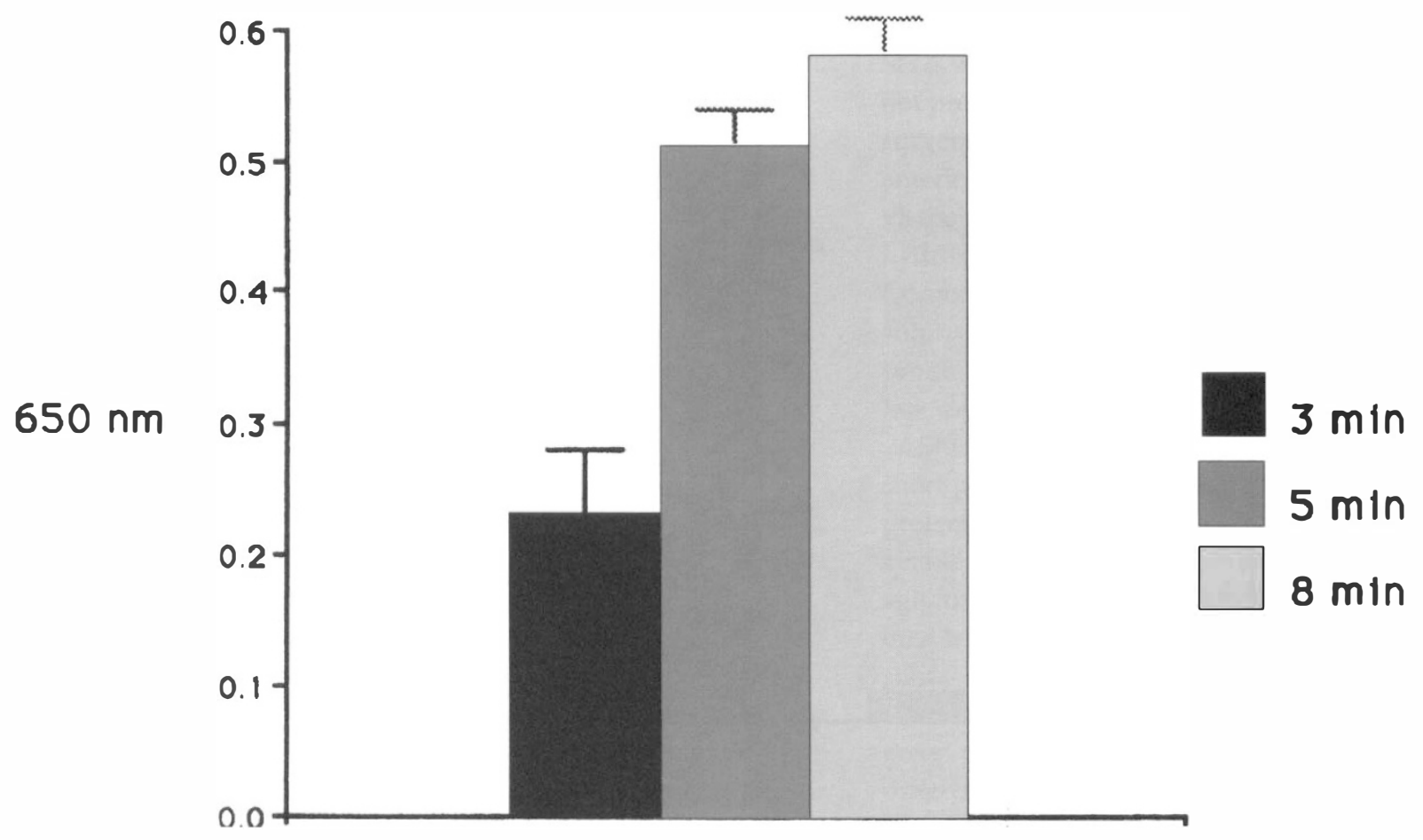

Fig. 2. Janus green photometry values (mean and standard deviation) following staining and elution of corneoscleral buttons from eyes that were subjected to irrigation and aspiration with balanced salt solution for 3,5 or $8 \mathrm{~min}$ before staining. 


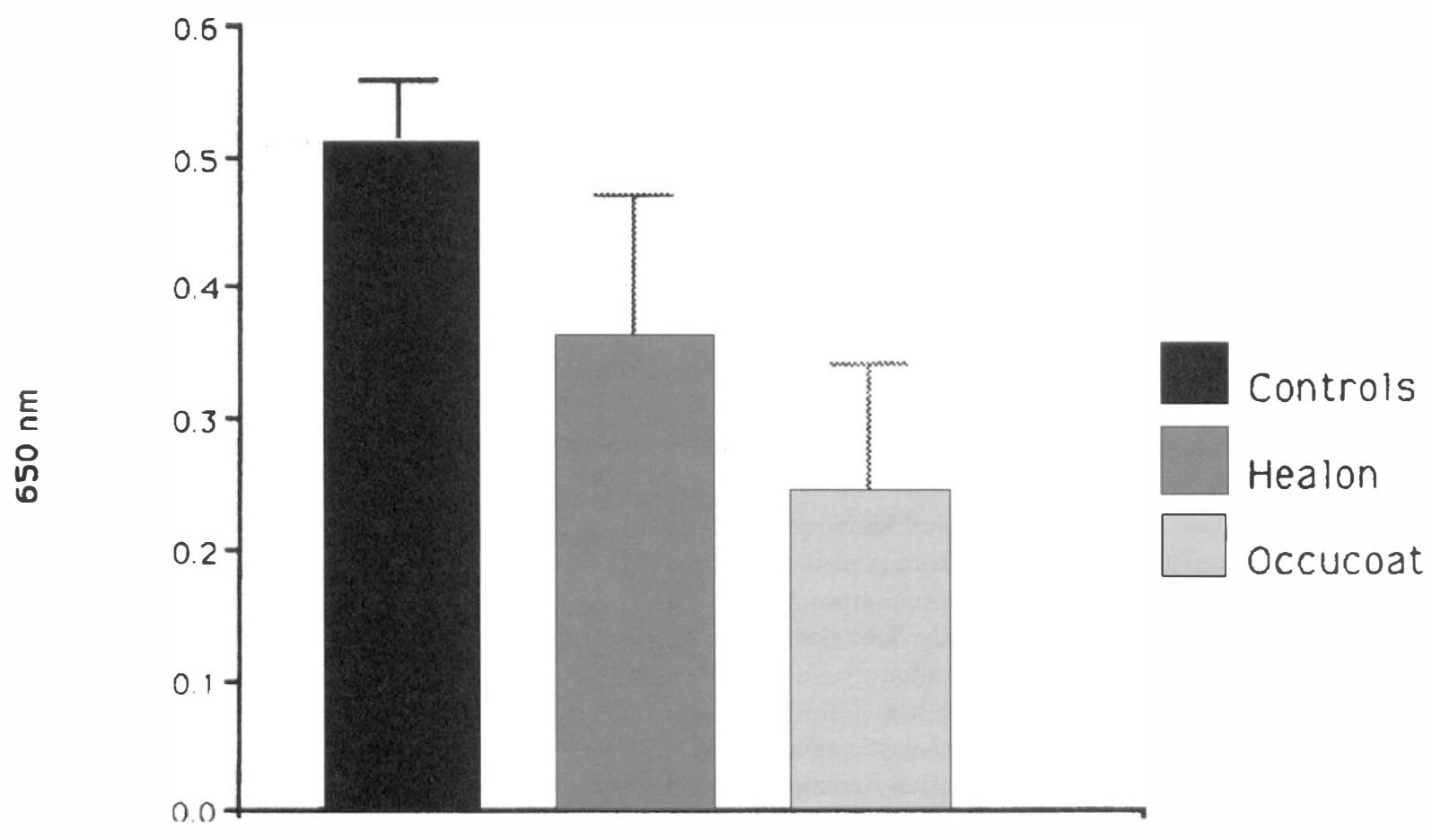

Fig. 3. Janus green photometry values (mean and standard deviation) following staining and elution of corneoscleral buttons from eyes where the anterior chamber was filled with SH (Healon) or HPMC (Occucoat) or neither (controls) prior to irrigation and aspiration for 5 min. Healon and Occucoat were used in paired right and left eyes of the same animals.

green photometric value of the 40 corneas protected with $\mathrm{SH}$ was 0.366 , corresponding to an average of $30.7 \%$ endothelial damage. The mean Janus green photometric value of the 40 corneas protected with HPMC was 0.24 , corresponding to an average of $20.5 \%$ endothelial damage. Both SH ( $p<0.01$, Student's $t$-test) and HPMC $(p<0.001$, Student's $t$-test) significantly protected the corneal endothelium from I\&A-induced damage compared with controls (Fig. 3). Of the two substances tested, HPMC afforded better protection to the corneal endothelium than $\mathrm{SH}(p<0.001$, Student's paired $t$-test).

\section{Discussion}

In the Janus green photometric technique the permeability of the cell and nuclear membranes for the vital stain Janus green is used as a marker for cell damage and cell death. This technique is simple and easy to use, independent of the user's experience and requires only standard laboratory equipment (photomicroscope, spectrophotometer). ${ }^{9-12}$ Pharmakakis et al. ${ }^{10}$ demonstrated that the Janus green photometric technique is an accurate method for determining endothelial damage, and can successfully replace the method of cell counting to evaluate the percentage of damaged endothelial cells.

This study established that I\&A can produce significant damage to the corneal endothelium of enucleated porcine eyes processed within $150 \mathrm{~min}$ of enucleation. The amount of endothelial damage as assessed by the Janus green photometry technique seems unduly high for the $5 \mathrm{~min}$ of I\&A but could be due the time interval of $60-150 \mathrm{~min}$ between enucleation and performing the test. The fact that these were postmortem eyes that were transported on ice (over a short distance) and brought to room temperature for the study, could have stressed the endothelium. Further, all eyes were hypotonus post-mortem and had to be clamped to bring the pressure up to $15-18 \mathrm{mmHg}$. This could also have contributed to the endothelial stress. These factors may have rendered the endothelium more vulnerable to irrigation-induced damage and could account for the high degree of endothelial damage observed in this study. It is possible that Tween-20 may have induced some degree of further endothelial damage, following I\&A, but this was not found to be significant. Any damage induced by the wash solutions or procedure would be applicable to both test groups and controls. Since right and left eyes of the same animal were used for comparison and as controls the comparative protection afforded by the two agents tested is still significant. Both $\mathrm{SH}$ and HPMC protected the endothelium against I\&A damage but HPMC was significantly more effective. It is of interest to note that, compared with $\mathrm{SH}, \mathrm{HPMC}$ was more difficult to rinse from the corneal endothelial surface. In order to make a valid comparison between $\mathrm{SH}$ and HPMC, Tween-20 had to be added to the rinsing solution (BSS) and the rinsing time increased to $3 \mathrm{~min}$ instead of the 2 min described in the original procedure. ${ }^{9}$ The affinity of HPMC for the endothelial surface is most likely responsible for the greater protective effect observed in this study.

The mechanism of endothelial protection by viscoelastic solutions is poorly understood. While solutions such as HPMC and chondroitin sulphate-sodium hyaluronate produce a clinically visible 
endothelial coating which protects the cornea in the short term post-operatively, it is not clear, in the long term, whether the presence of this coating or the particular molecules themselves alter the endothelial microenvironment.

The endothelium has known hyaluronic acid receptors as well as a normal coating of hyaluronic acid molecules which is easily washed away by endothelial irrigation. ${ }^{13,14}$ Interestingly, studies have shown that the endothelial coating of hyaluronic acid can be replaced by similar molecules placed in the anterior chamber. ${ }^{13}$ The significance of the normal hyaluronic coating to the endothelium and its health is uncertain.

The clinical relevance of this study is not known as it is difficult to extrapolate data obtained from postmortem eyes to the viable clinical situation. Simple irrigation for a brief period of time usually does not appear to cause endothelial damage in human endothelium in vivo. This communication has demonstrated the beneficial effect of viscoelastic agents in protecting the endothelium from irrigation damage in porcine eyes in vitro. Further research is needed to investigate the potential clinical importance of these findings.

We wish to thank Prof. Larry A. Donoso, Director of Research, Wills Eye Hospital, for his support and the late Leonard Foster for his technical help. A.A-B. was the Vision Express Fellow in Cornea and Contact Lenses at the University Hospital, Nottingham from July 1997 to December 1998.

\section{References}

1. Beesley, R, Olson RJ, Brady SE. The effects of prolonged phacoemulsification time on the corneal endothelium. Ann Ophthalmol 1986;18:216-22.

2. Craig MT, Olson RJ, Mamalis N, Olson RJ. Air bubble endothelial damage during phacoemulsification in human eye bank eyes: the protective effects of Healon and Viscoat. J Cataract Refract Surg 1990;16:597-602.
3. Monson MC, Tamura M, Mamalis N, Olson RJ, Olson RJ. Protective effects of Healon and Occucoat against air bubble damage during ultrasonic agitation of the anterior chamber. J Cataract Refract Surg 1991;17:613-6.

4. Glasser DB, Katz HR, Boyd JE, Langdon JD, Shobe SL, Peiffer RL. Protective effects of viscous solutions in phacoemulsification and traumatic lens implantation. Arch Ophthalmol 1989;107:1047-51.

5. Glasser DB, Osborn DC, Nordeen JF, Min YI. Endothelial protection and viscoelastic retention during phacoemulsification and intraocular lens implantation. Arch Ophthalmol 1991;109:1438-40.

6. Pedersen OO. Comparison of the protective effects of methylcellulose and sodium hyaluronate on corneal swelling following phacoemulsification of senile cataracts. J Cataract Refract Surg 1990;16:594-6.

7. Assia EI, Apple DJ, Lim ES, Morgan RC, Tsai JC. Removal of viscoelastic materials after experimental cataract surgery in vitro. J Cataract Refract Surg 1992;18:3-6.

8. Binder PS, Sternberg H, Wickham MG, Worthen DM. Corneal endothelial damage associated with phacoemulsification. Am J Ophthalmol 1976;82:48-52.

9. Hartmann C, Rieck P. A new test for endothelial viability: the Janus green photometry technique. Arch Ophthalmol 1989;107:1511-5.

10. Pharmakakis N, Hartmann C, Hilgers RD, Bergmann L, Koliopoulos I. Correlation study of two methods for evaluating corneal endothelial damage in vitro: the Janus green photometry technique versus cell counting. Ophthalmic Res 1995;27:67-73.

11. Pels E, Nuyts RMMA, Breebart AC, Harmann C. Rapid quantitative assays for corneal endothelial cell viability in vitro. Cornea 1993;12:289-94.

12. Baumann G, Fries U, Schnaudigel O-E. Corneal endothelial viability after long-term storage at $+4^{\circ} \mathrm{C}$ in Optisol. Ophthalmologe 1994;91:624-7.

13. Madsen K, Schenholm M, Jahnke G, Tengblad A. Hyaluronate binding to intact corneas and cultured endothelial cells. Invest Ophthalmol Vis Sci 1989;30:2132-7.

14. Madsen K, Stenevi U, Apple DJ, Harfstrand A. Histochemical and receptor binding sites of hyaluronic acid on corneal endothelium. Ophthalmic Practice 1989;7:1-8. 